\title{
Hip sonography update. Quality-management, catastrophes - tips and tricks
}

\author{
Reinhard Graf, Mohammad Mohajer, Florian Plattner
}

Orthopädie LKH-Stolzalpe, Austria

\begin{abstract}
Aim: Hip sonography is today considered to be the diagnostic standard tool for developmental dysplasia of the hip (DDH) diagnosis. In Germany special commissions control the quality of the sonograms according to checklists to keep the standard high and to avoid over- and under - treatment. This paper lists tips and tricks to avoid typical mistakes according to these results. Material and method: In 8 German countries the quality of sonograms used to diagnose DDH was checked according to special checklists. An additional 250 medical doctors (pediatricians, radiologists, orthopaedic surgeons) were asked to classify 4 sonograms by measuring and typing. From the same group the projection (standing left, standing right, horizontal-cranial left, horizontal-cranial right) was checked according to the correct diagnosis in the shortest time. Results: In $1.6 \%-43.7 \%$ the licence for hip sonography was withdrawn by the German commission. The best projection with the lowest rate of wrong interpretation was the "standing right"-projection. Most of the mistakes were made because of a wrong anatomical identification and usability check. Conclusion: Hip sonography must be trained by authorized teachers. The quality of the sonograms must be controlled according to checklists. The bedside teaching without control leads to a systematic teaching of mistakes and finally catastrophes.
\end{abstract}

Keywords: DDH-diagnosis, hip sonography, dysplasia.

\section{Introduction}

Since the first publication in 1980 [1] about hip sonography the method has been continuously developed [2]. Because the method is standardized and reproducible, it allows the classification of the baby hip joint in accordance with their age, the maturity of the bony roof, as well as the hyaline cartilage roof. This special technique has even accepted by the health systems in different countries [3]. Because of the high incidence of developmental dysplasia of the hip (DDH) in central Europe, a general screening of all newborn was introduced in Austria (1991), Switzerland (1995) and Germany (1996).

Received 15.08.2013 Accepted 29.08.2013

Med Ultrason

2013, Vol. 15, No 4, 299-303

Corresponding author: Reinhard Graf

Hagersiedlung 7

A-8850 Murau-Stolzalpe, Austria

E-mail: reinhardgraf@gmx.at
The rate of open reductions, acetabuloplasty and head necrosis were reduced dramatically [4]. Depending on different health systems, hip sonography is performed as a local screening or a risk screening [5].

In Germany special quality commissions from the Bundesärztlichen Kassenvereinigungen control the quality of the sonograms according to a special system to preserve the quality of the hip sonography and to avoid wrong diagnoses [6].

The aim of this paper is to demonstrate typical mistakes during examination and to give a handout with tips and tricks to avoid catastrophes for the babies.

\section{The quality management control}

1. The quality management control commission from Germany checked in 2011 in 8 states the quality of hip sonograms using the "Graf technique". According to the checklists, in 1.6-43.7 \% cases the license for hip sonography was withdrawn because of poor quality diagnoses [7]. 


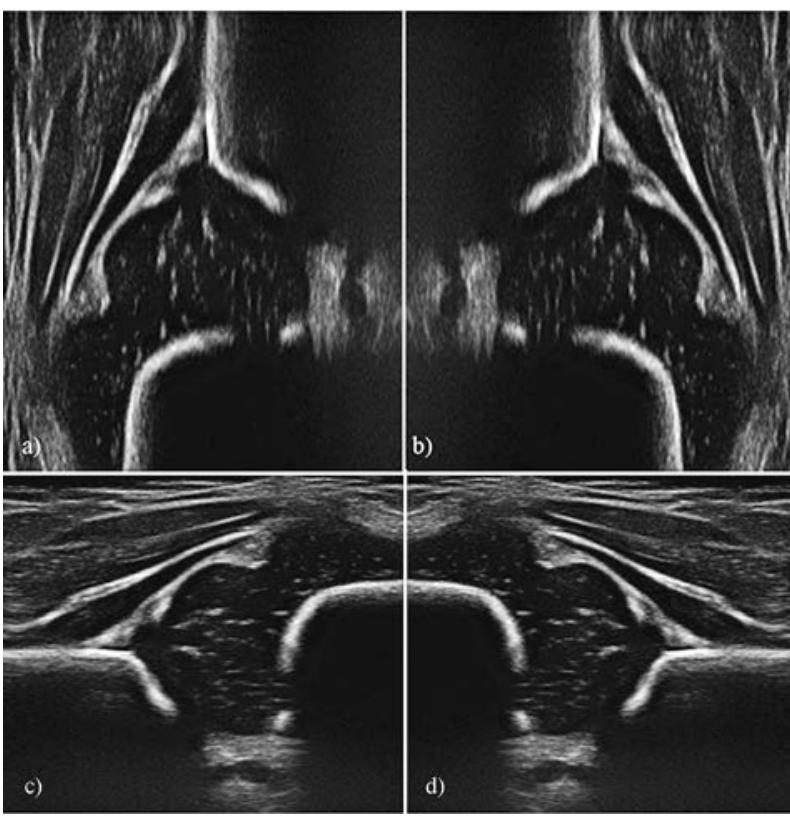

Fig 1. Possible projections of hip sonogramms: a) standing-up right position, b) standing-up left position c) horizontal-cranial right position d) horizontal-cranial left position. From best to worst: a), d), b), c).

2. In refresher courses held in 2011 in Austria and Germany 250 medical doctors (orthopaedic surgeons, paediatricians and radiologists) who performed hip sonography had to classify 4 sonograms. The results and the typical mistakes were as following: $28 \%$ passed; $36 \%$ of errors occurred in the measurement lines, and $64 \%$ had the wrong anatomical identification or in not visible landmarks.

3. In another refresher course (1990, Stolzalpe) 60 participants divided in mixed groups, received sheets with 4 sonograms from the same hip joint in different projections in order to establish which one ensured the correct measurement and typing in the shortest time. Also, we checked if there are differences between orthopaedic surgeons, paediatricians, radiologists and sonographers in interpretation of sonograms?

Generally the reasons for a wrong diagnosis were: 1) a poor examination technique with tilting effects which may lead to overdiagnosis (fig 1); 2) a wrong anatomical identification of the sonographic echos; 3 ) no usability check.

The best projection of the hip joint for the interpretation was in the standing up right position, such as the right hip joint in a radiogram. The worst was the horizontal-cranial left position. There were no differences between the groups and the results were independent to the participants' specialities.

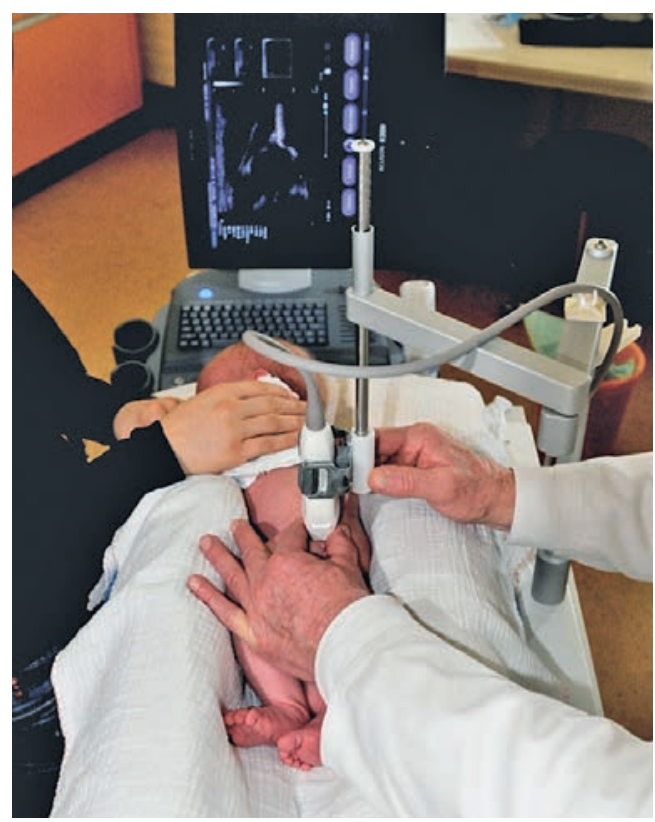

Fig 2. Examination of the left hip joint with cradle and probe guide system to avoid tilting effects which may lead to overdiagnosis.

Explanation: The perception of pictures in variable projections depends on unequal activities of the brain hemispheres.

\section{How to avoid mistakes?}

\section{Technical equipment:}

- 5-7.5 or more $\mathrm{MHz}$ linear probes, no trapezoid or sector probes

- tilted probes with consecutive oblique ultrasound may lead to overdiagnoses

- a baby positioning device and a probe guide system (SonoFix positioning device and SonoGuide probe guiding system) should be used for a routine handling to avoid tilting effects (fig 2).

\section{Examination of the baby:}

- at first look always to the lower limb of the os ilium (!) and not to the plane!

- after the lower limb of the os ilium is fixed rotate the probe into the correct plane (=standard plane)

- when the lower limb disappears because of the rotation, look for the lower limb again. Using the probe guide system and the cradle, all the other structures such as chondroosseous border, labrum, etc. will automatically appear.

\section{Checklist:}

After the sonographic examination, the sonogram must be checked in a certain way. 
Anatomical identification (checklist 1) (fig 3)

All the anatomic structures from checklist 1 must be visible. If only one of the structures is unclear or cannot be identified, the sonogram is useless. The examiner is always on the "safe side" when all the structures are identified. In this way automatically typical misinterpretations or confusions such as mixing up of labrum with synovial fold or tilting effects which lead to overdiagnosis, are avoided.

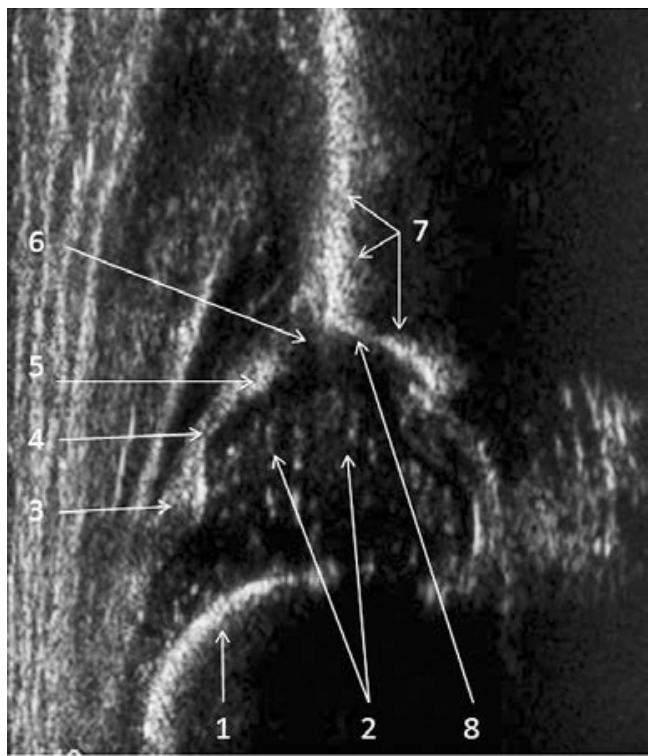

Fig 3. Checklist 1: Anatomical identification: 1 - chondroosseous border, 2 - femoral head, 3 - synovial fold, 4 - joint capsule, 5 - labrum, 6 cartilagineous roof, 7 - bony roof, 8 - bony rim (concavity-convexity).
Usability check (checklist 2) (fig 4)

After the anatomical identification the sonogram must be checked if it is in the correct plane:

- does it go through the middle of the acetabulum (the lower limb of the os ilium in the fossa acetabuli must be visible)?

- does it go through the middle of the acetabular roof (the os ilium contur must be straight or parallel to the probe)?

- are tilting or oblique planes avoided (is the labrum visible?)?

A plane needs 3 landmarks, therefore checklist 2: Lower limb-plane- labrum

Note: In the case of DDH the femoral head leaves the standard plane and moves in to the dorsal-cranial direction. It means, if according to the anatomical identification a femoral head dislocation is diagnosed (Type 3 or Type 4), the measurement is not needed or possible. In this case the usability checks are not needed because the head is often not in the plane (fig 5).

4. Measurement mistakes (fig 6):

- only sonograms in the standard plane are accepted to be measured

- the bone roof line is in contact with the bone and does not go through the bony rim automatically

- the cartilage roof line comes from the bony rim (this is a turning point from the concavity to the convexity!) and not from the crossing point of the bony roof line and the acetabulum roof line. It goes through the middle of the labrum and not to the top of the labrum!

5. Classification of instability, elastic whipping and typically mistakes:

(All types are listed in the "Sonometer" - fig 7)
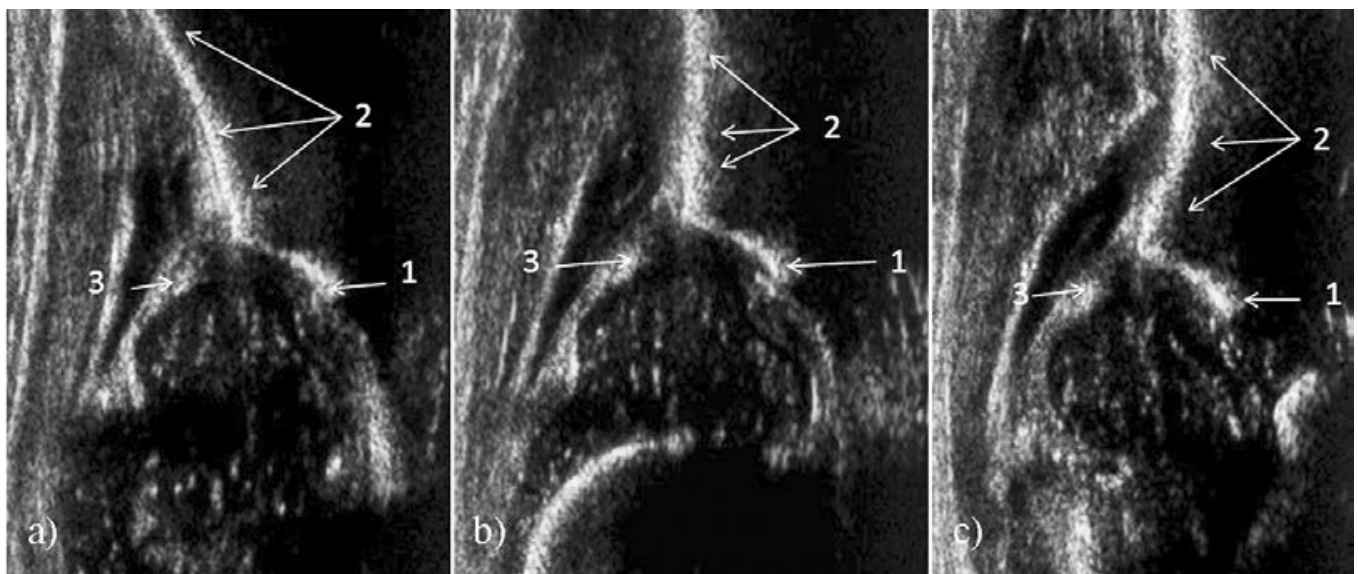

Fig 4. Checklist 2: a) anterior plane; b) standard plane; c) posterior plane. 1. lower limb of os ilium; 2."plane" 3. Labrum. 


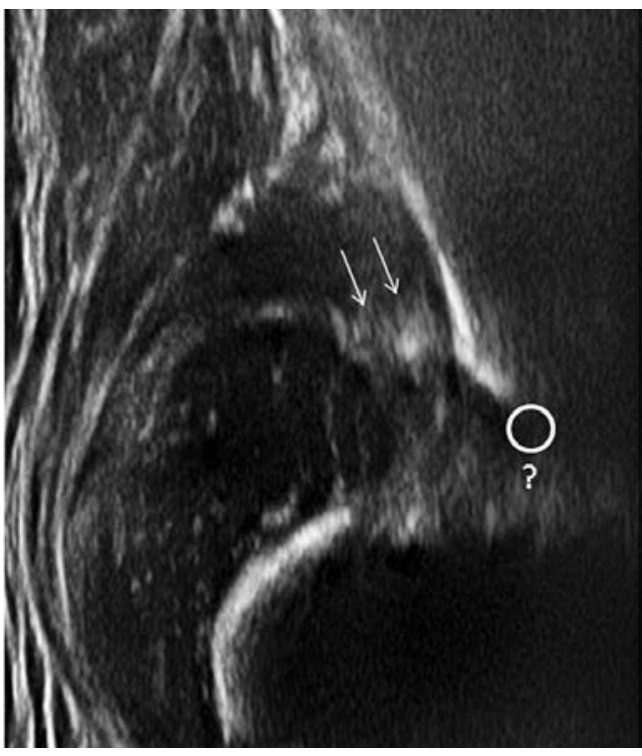

Fig 5. DDH Type 4: The femural head is dislocated and therefore is not in the standard plane. The lower limb is not visible (?). Typing (diagnosis) is made by morphology, not by measurement!

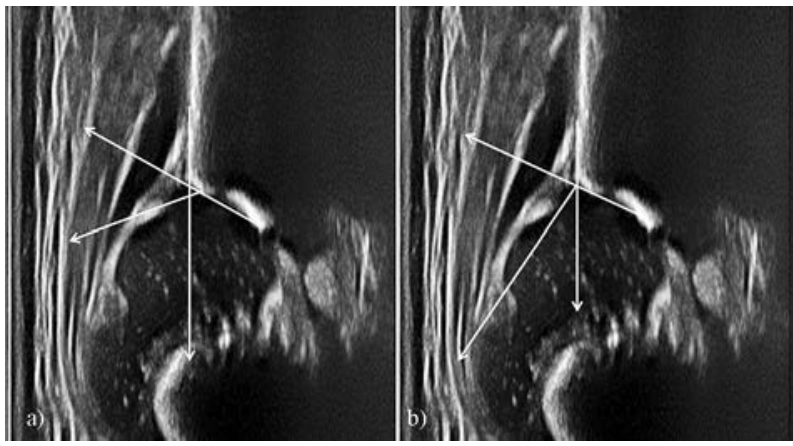

Fig 6. Measurement: a) correct measurement; b) incorrect measurement: the bony roof line „cut" the lower limb of os ilium. The cartilage roof line comes from the crossing point of the bony roof- and the base line instead of the bony rim(!) and goes through the synovial fold instead of the middle of the labrum. 1 - bony roof line, 2 - base line, 3 - cartilage roof line.

Note: Alpha and Beta angles must be measured_

- for the Beta angle the cartilage roof line is needed. For the cartilage roof line it is necessary to identify the bony rim and the labrum. Lack of documentation of the Beta-value means the lack of correct documentation of the bony rim and the labrum. This may be a legal problem in the case of a wrong diagnosis.

- Alpha and Beta angles values are to be measured to distinguish "pathological" instability from the "harmless" movements, which are called "elastic whipping".

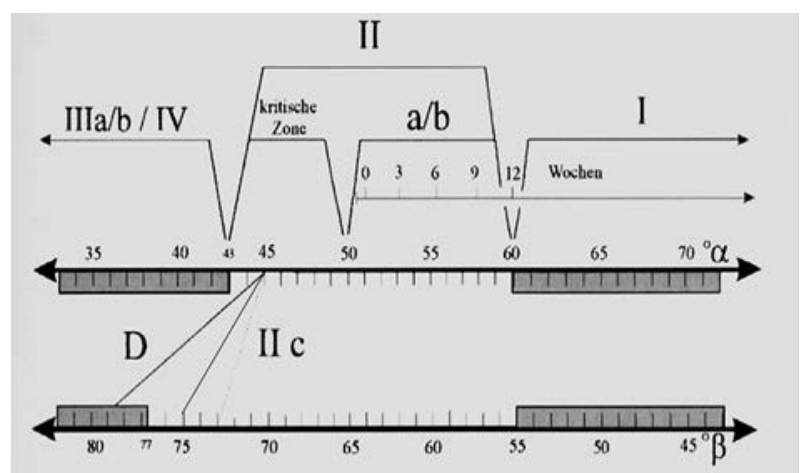

Fig 7. Sonometer with subdivision of the types. E.g. Typ 2c and type D to measure instability and seperate it from ,elastic wipping".
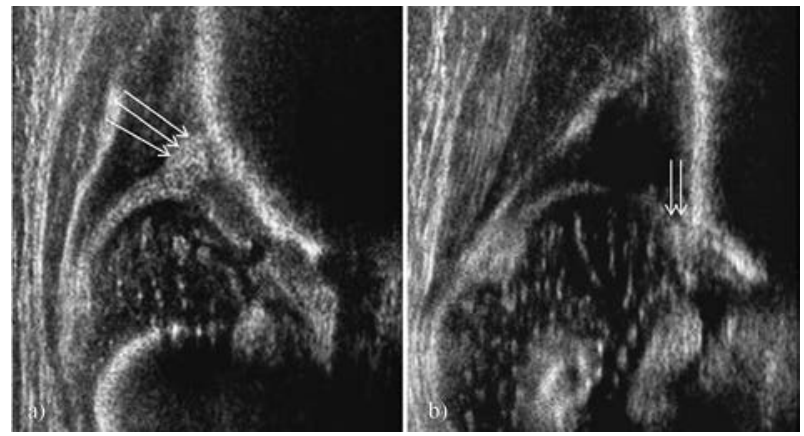

Fig 8. The course of the perichondrium makes sonographically the difference between Type 3 and Type 4: a) perichondrium goes upwards - Type 3 ; b) perichondrium goes horizontal Type 4.

In this way instabilities can be objectified and independent from examiners experience [3].

Sonographic techniques which are not able to distinguish an elastic whipping from a pathological instability can not reach the standard which is needed today [8]. Decentred joints are always unstable and out of discussion. The border is important: Is it, or is it not! (Type IIc stable or Type IIc unstable).

- Type IIa: these joints are younger than 3 months. They look like a dysplasia, but according to their age they are acceptable: They are "physiological immature" and there is no need for a treatment. In risk-babies a control is recommended.

- Type IIc: it is the so called "critical range" and is considered to be a sever dysplasia; it needs treatment in any way, independent from the age.

- Type IIb: these are dysplastic joints which are older than 4 months. Usually treatment is required. 
The sonographic difference between Type III and Type IV (fig 8) is made by the course of the perichondrium and not by the position of the labrum! The perichondrium shows indirectly where the cartilage roof is if is pressed upwards or downwards: the perichondrium contour rise upwards- Type 3; the perichondrium contour runs horizontal or makes a concavity- Type 4 . The reposition obstacle of the femoral head is due to the deformed cartilage roof and not the labrum!

\section{Discussion and conclusion}

Hip sonography has spread worldwide. The inconsistent hip sonography techniques used in different countries and centres are the main cause of ongoing discussions regarding hip sonography and its reliability. In the German speaking countries there are strict rules for quality management of the sonograms. A special education and training program for medical doctors is established. Hip sonography must be done in a reproducible and a standardized way, independent from the experience and the skill of the examiner. Hip sonography is always a dynamic examination: the joint is checked as in a tomogram. To make it comparable and reproducible, a "standard plane" is to be used for the diagnosis. Additionally a stress test ("dynamic examination") in borderline cases (Type IIc or worse) is recommended to verify the instability by measurement and to separate it from harmless "elastic whipping". Typical mistakes can be avoided if checklist 1 and checklist 2 are used (such as checklists in an aeroplane before takeoff).

Hip sonography using this technique has reduced the conservative treatment rate and avoided overtreatment as well as operations and consequently femoral head necrosis. Long term results are impressive [4,9]. According to the experience in Austria, Switzerland and Germany, the examiners must be trained by authorized teachers in special training courses. Bedside teaching from non authorized people may lead to a systematic teaching of mistakes! Whether hip sonography is done as a screening or only for risk babies depends on the health system in different countries as well as the incidents of the DDH in different areas.

\section{References}

1. Graf R. The diagnosis of congenital hip-joint dislocation by the ultrasound Combound treatment. Ach Orthop Trauma Surg 1980; 97: 117-133.

2. Graf R. Sonographie der Säuglingshüfte und therapeutische Konsequenzen. Thieme Stuttgart, New York, 2010.

3. Graf R. Hip sonography: Diagnosis and Managment of Infant Hip Dysplasia. Springer 2006.

4. Grill F, Müller D. Ergebnisse des Hüftultraschallscreenings in Österreich. Orthopade 1997; 26: 25-32.

5. Von Kries R, Ihme N, Oberle D, et al. Effect of ultrasound screening on the rate of first operative procedures for developmental hip dysplasia in Germany. Lancet 2003; 362: 1883-1887.

6. Kassenärztliche Bundesvereinigung. Ultraschallvereinbarung gemäss $\$ 135$ Absatz 2. SGBV. Berlin 2012.

7. Tschauner C, Matthissen HD. Hip sonography with Grafmethode in Newborns: Checklists help to avoid mistakes. OUB 2012; 1: 7-8.

8. Harcke HD, Grissom LE. Sonographic evaluation of the infant hip. Semin Ultrasound 1986; 7: 331-338.

9. Tschauner C, Fürntrath F, Radl R, Berghold A, Schwantzer G. Improved therapeutic outcome of decentered hip joints by early sonographic diagnosis using the Graf technique Results of a single center retrospective cohort study of 3 decades (1978-2007). OUB 2012; 1: 390-393. 